\title{
CARACTERÍSTICAS DE LOS TUTORES PARA EL TRABAJADOR DE LA GENERACIÓN "Y" PERUANO: IMAGEN EN BASE A UN ESTUDIO DE CAMPO
}

\author{
Luis Alfonso Cruz Álvarez \\ Universidad Internacional Iberoamericana (México) \\ hagen78@hotmail.com $\cdot$ https://orcid.org/0000-0001-8123-0729 \\ Sonia Rodríguez Fernández \\ Universidad de Granada (España) \\ soniarf@ugr.es $· \underline{\text { https://orcid.org/0000-0002-6883-8884 }}$
}

\begin{abstract}
Resumen. El presente artículo es parte de una investigación doctoral la cual pretende aportar en los campos del conocimiento de la pedagogía y la gestión de personas, investigando la percepción que tienen los trabajadores peruanos de la generación "Y" de lo que debe ser la figura de un líder que tome el papel de Tutor y Orientador en un ambiente laboral. Para esta investigación se ha realizado una revisión de la literatura sobre el tema y adicionalmente un estudio de campo utilizando una herramienta de recolección de datos diseñada ad hoc. Esta herramienta ha sido validada por un grupo de expertos cuyos resultados fueron analizados mediante el estadístico W de Kendall para verificar la concordancia interna de las preguntas. En general la herramienta tuvo un índice de Kendall (W) de 0.572 y un nivel de significancia (Sig) de 0.032, lo cual se concluye que es una herramienta de un adecuado nivel de concordancia entre los expertos, así como que la concordancia entre ellos no se debe al azar. Luego de la validación, hemos aplicado la encuesta a un grupo de 149 trabajadores de la generación "Y" peruanos de distintos campos laborales. Los resultados fueron sometidos a análisis no paramétricos de diferencias de medias del tipo TStudent y ANOVA, concluyendo que los trabajadores de la generación "Y" con educación superior tienen una predisposición favorable a la figura del tutor en el trabajo. Así mismo, esta figura presenta determinadas características que podremos analizar más al detalle en la discusión de resultados. Es importante recalcar que estos resultados están en línea con otras investigaciones similares realizadas.

Palabras clave: generación "Y”, innovación, orientación y tutoría
\end{abstract}

\section{CHARACTERISTICS OF THE TUTORS FOR THE WORKER OF THE PERUVIAN "Y" GENERATION: IMAGE BASED ON A FIELD STUDY}

\begin{abstract}
This article aims to contribute in the fields of knowledge's pedagogy and people management by investigating the perception that Peruvian millennial workers have of what should be the figure of a leader who takes the role of Mentor and Counselor in an environment labor. For this research, a review of
\end{abstract}


the literature on the subject has been carried out and, in addition, a field study using a data collection tool designed ad hoc. This tool has been validated by a group of experts whose results were analyzed by means of two analyzes, one of means of value of each question and then by a statistician to verify the internal concordance of the questions, W of Kendall. In general, the tool had a Kendall index (W) of 0.572 and a level of significance ( $\mathrm{Sig}$ ) of 0.032 , which concludes that it is a tool of high level of agreement among the experts. After this validation we applied the survey to a group of 149 Peruvian millennial workers from different labor fields. The results were subjected to nonparametric analysis of mean differences of both the T-Student and ANOVA types, with the main conclusion that millennial workers with higher education have a favorable predisposition to the figure of the mentor at work. Also this figure presents certain characteristics that we can analyze more in detail in the discussion of results. It is important to emphasize that these results are in line with other similar investigations carried out.

Keywords: Millennials, innovation, mentoring and couseling.

\section{Introducción}

Los trabajadores de la generación "Y" son aquellos trabajadores que han nacido entre los años 1982 a 2002 (Epstein y Hershatter,2006; Howe y Strauss, 2000) y que presentan rasgos como los de haber crecido en tiempos prósperos, tener grandes expectativas y buscar sentido en el trabajo que realizan (Howe y Strauss, 2000). Asimismo, Boschma y Groen (2008) agregan a que dichos trabajadores se les reconocen como los de la sociedad de la información, conectados todo el tiempo, en cualquier lugar y a cualquier hora. Los investigadores peruanos Castro y Yamada (2010) coinciden con lo dicho sobre los trabajadores de la generación "Y" pues en el contexto peruano en que crecieron este tipo de trabajadores, se caracterizó por un periodo de crecimiento económico, por lo que sus necesidades básicas estuvieron cubiertas con lo que generó una actitud un tanto despreocupada con la realidad.

La consultora Deloitte (2015) define algunas características de los trabajadores peruanos de la generación "Y": son hiperconectados, tienden a una alta rotación laboral, son informales en el trato, participativos, buscadores de balance en sus vidas, gustan del aprendizaje continuo, buscan su autodesarrollo y son propensos a recibir mentoría. La consultora también indica que le dan una importancia marcada al tipo de liderazgo que desean tener y es por ello que piden un líder que sea: una fuente de inspiración, tenga habilidades interpersonales, visión, pasión y entusiasmo, sepa tomar decisiones, tenga pensamiento estratégico, que transmita comodidad, de flexibilidad, sepa dar retroalimentación y que esté orientado a las personas, es decir, lo vean como un mentor (Deloitte, 2015 y Pricewaterhouse, 2014). En esta misma línea la consultora EP|Roland (2014) indica que: "Los jefes deben actuar como guías más allá de la vida profesional y crear desafíos constantes" (ER|Roland, 2014 p.11) reforzando la imagen de mentor o tutor que este tipo de trabajadores buscan.

Tapscott (2009, citado por Gonzáles, 2011) define algunas líneas rectoras que cualquier trabajador de la generación "Y" desea encontrar en un ambiente laboral ideal:

a) Libertad para trabajar cuándo y dónde quieran, es decir, flexibilidad horaria.

b) Personalización, pues reclaman no ser tratados como masa sino como individuos.

c) Información abierta y fluida entre los compañeros y con los líderes.

d) Integridad, porque estos trabajadores esperan colaborar con una empresa que sea transparente, honesta y considerada con su entorno.

e) Colaboración, trabajando con otros para alcanzar los objetivos. 
f) Disfrutar el trabajo que hacen, por lo que los ambientes laborales deben ser entretenidos y agradables.

Adicionalmente Gonzales (2011) refuerza el tema de la mentoría pues explica que estos trabajadores buscan alguien que los guie, los aconseje, sea un referente, una persona íntegra y por la cual sientan confianza para desarrollarse.

Redondeando las ideas presentadas, de Begazo y Fernández (2015) vislumbran que este grupo acentuará su deseo de acceso inmediato a la contribución de la organización, una constante y más detallada retroalimentación de sus superiores, mayor equilibrio entre vida personal y vida laboral y espacios para retribuir a la comunidad mediante trabajos filantrópicos.

En conclusión, apreciamos que los principales rasgos que resaltan a este grupo de generacional de trabajadores son: estar hiperconectados, ser participativos, buscar balance entre la vida y el trabajo, aprendizaje continuo, autodesarrollo y una búsqueda por ser guiados. Por lo tanto, es una generación que está a la expectativa de su desarrollo por medio de la retroalimentación y de jefe o líderes que los sepan guiar.

El otro eje que maneja la investigación es la tutoría y orientación y en este punto, aclaramos que la investigación tomará el enfoque de la tutoría y orientación en ambientes de trabajo, aunque este concepto venga del ámbito de la pedagogía.

Para comenzar, debemos precisar qué tipo de educación se da en las empresas. Para Vásquez (1998, citado por Carrasco, 2014) es una educación no formal pues está enfocada a desarrollar una o varias funciones en el trabajo.

Esta educación tiene un componente de aprendizaje que para Agrys y Schon (1960, citados por Carrasco, 2014) es un ciclo doble, donde se explota el conocimiento existente y se generan nuevos conocimientos, con lo que se permite pensar de otras maneras. En palabras de Carrasco (2014) la educación y el aprendizaje en la empresa le dan a esta la capacidad de: "ser capaz, como organización, tanto de sostener el conocimiento útil y conocido como de romper esquemas y encontrar nuevas y mejores formas de realizar el trabajo" (Carrasco, 2014 p.32). Como hemos apreciado la educación en el ámbito laboral está guiada hacia el adiestramiento en el puesto de trabajo a un nivel inicial, pero también hacia la generación de nuevo conocimiento, lo cual implica la existencia de competencias laborales que produzcan esa acción y además que este proceso se potencie mediante la figura de un tutor que guíe este proceso.

La tutoría y orientación, en el mundo empresarial, está dentro de las funciones de los departamentos de recursos humanos y específicamente en dos de sus procesos; en la inducción cuyo objetivo es que los nuevos trabajadores conozcan y socialicen en la empresa y en la evaluación del desempeño, en el momento en que se da la retroalimentación y se crean acciones de seguimiento para las acciones de mejora (Cannice, Koontz y Weihrich, 2012). Aunque los autores ubican este proceso en las funciones antes mencionadas, en la realidad, la tutoría y orientación se da en dos prácticas laborales cotidiana: el coaching y la mentoría. El coaching, es un proceso donde interviene el trabajador y el coach o consejero en donde, en sesiones individuales, se tratan temas que implican cambios profundos en la vida profesional del trabajador. Por otra parte, la mentoría es una metodología de aprendizaje en donde el mentor y el aprendiz tienen conversaciones que están claramente orientadas a tener un resultado en la función del puesto. Es un pasar conocimiento desde la experiencia (Espinosa, 2011, Serrat, 2010). 
Con ambas técnicas se pretende que el trabajador tenga un aprendizaje estratégico o significativo, el cual relaciona la actividad, el sujeto y el contexto. Un elemento fundamental, dentro de la adquisición de este tipo de aprendizaje, es el tutor laboral que para Schalk (2005) es:

Quien hace sentir al trabajador parte de un universo nuevo y altamente motivante, es quien lo integra e incorpora y para ello es necesario: conocer al trabajador, entregar sugerencias respecto a sus objetivos y necesidades de inscribirse a una actividad determinada. (Schalk, 2005 p.82)

Reforzando la propuesta anterior, Coll, Mauri y Onrubia (2008) hablan de la Zona de Desarrollo Próximo (ZDP) como una zona donde el capacitador y el aprendiz tienen una interacción en donde el capacitador, como experto, favorece en el aprendiz un funcionamiento interpsicológico que es fundamental para construir conocimiento. De esta forma el papel del tutor laboral es indispensable para que el aprendiz interiorice el conocimiento.

Las investigaciones de Brcic y Mihelic (2015) apuntan a que la generación "Y" tiene una disposición a recibir más mentoría por lo que recomiendan que las organizaciones pongan mayor énfasis en propiciar de manera sistemática, relaciones de mentoría intergeneracional. En línea con lo visto por estos investigadores, la encuesta Great Place to Work (2013) indica que los trabajadores de la generación "Y" piden a sus jefes comunicación y feedback con el fin de desarrollarse profesionalmente, moldeando así, un tipo de relación en el que lo líderes o jefes sean: "mentores o coaches" (Great Place to work, 2013 p. 6).

De toda la literatura revisada podemos concluir que, existe un punto de convergencia, en teoría, entre los conceptos de trabajadores de la generación "Y" y la tutoría y orientación. Este punto de convergencia apunta a que la generación "Y" presenta una tendencia a recibir de manera favorable a la Tutoría y Orientación, mediante la figura del Tutor. Pero ¿hay algún tipo de estudio que haya versado sobre la percepción que los trabajadores de la generación "Y" peruanos tienen sobre la figura que tiene que tener el tutor en ambientes laborales?

Revisando la literatura sobre la cuestión, un primer estudio es el de Dávila y Mitta (2015) que trató de identificar políticas y prácticas de retención para los trabajadores de la generación " $Y$ " en diferentes organizaciones peruanas para lo cual se dividió el estudio en dos partes; en la primera se estudió los requerimiento y expectativas laborales de los jóvenes de la generación "Y" y en la segunda parte se identificó las políticas y prácticas de retención actuales de los departamentos de recursos humanos de las organizaciones que han participado en el estudio. Para ello se construyó una encuesta ad hoc dirigido a estudiantes del último año de la universidad, basado en el perfil de la generación "Y" de los jóvenes norteamericanos y luego una entrevista ad hoc a los encargados de recursos humanos para saber su opinión sobre los jóvenes de la generación "Y". Los resultados de este estudio demuestran una brecha entre lo que las empresas esperan de los jóvenes de la generación "Y" en el campo laboral, frente a lo que ellos son en realidad. Pero no es una brecha negativa sino más bien una brecha de expectativas, es decir, que ambas partes esperan un pivote para desarrollar ese potencial. Es de especial interés que las empresas vean que los jóvenes de la generación "Y" tienen capacidad de innovación, ideas nuevas y aporten perspectiva diferente del mundo y que los mismos se vean como flexibles con las reglas, pero no aún tan innovadores como desearían. Así cabe resaltar que ellos piden siempre 10 
una constante retroalimentación de lo que hacen, lo que denota una necesidad de orientación y tutoría para justamente explotar ese potencial innovador, así como de otras competencias.

En otro estudio sobre el uso de la mentoría o el coaching para retener a los trabajadores de la generación "Y" y desarrollar sus potencialidades fue hecho por Newell (2015) quien estudia una propuesta de formación de los trabajadores de la generación "Y" mediante modalidades de social learning para lo cual realizó el estudio con los trabajadores de una empresa de telecomunicaciones en Perú. Para ello se llevaron a cabo varios focus group sobre la forma de generar el contenido necesario para estas nuevas plataformas de aprendizaje, surgiendo como principal conclusión que un aprendizaje dirigido a trabajadores de la generación " $Y$ " tendrá mayor impacto en tanto es construido con recursos internos lo que es lo mismo que aprovechar el conocimiento de colaboradores expertos y referentes en temas específicos, los cuales se convertirían en mentores de este aprendizaje.

Otro caso de estudio sobre la mentoría, aunque fuera del ámbito peruano, fue el programa de desarrollo del trabajador en ambientes laborales españoles realizado por Grande y Nuñez-Cacho (2012). La investigación plantea la figura de la mentoría como la asignación de un pupilo, que es un empleado con posibilidad de promoción y mejora en la empresa, a un mentor que es otro empleado con una experiencia y conocimiento profundo de la organización. Esta parte se define como crítica puesto que el éxito de este proceso dependerá mucho de la afinidad, confianza y buen clima en la relación entre pupilo y mentor. La investigación se realizó en dos partes, primero una encuesta ad hoc en donde se recogía información sobre productividad y crecimiento de la empresa basados en programas de mentoría. Esta se llevó a cabo en empresas españolas de distintos sectores y en donde la media de los trabajadores rondaba los 35 años. En la segunda parte, los resultados fueron luego sometidos a un modelo de ecuaciones estructurales que tenía como variable dependiente al crecimiento de la empresa. La conclusión a la que llegan dichos autores es:

Cuando la organización emplea un mentor para que el empleado se desarrolle como trabajador y persona, los procesos son programados por la organización (mentoring formal), incluyendo objetivos en el programa, se utiliza un mentor externo cuyo perfil ha estudiado detenidamente el proceso, se desarrolla a largo plazo con participantes satisfechos con la carrera que llevan en la organización y comprometido con la relación del mentoring; entonces se mejorará el rendimiento del capital humano. (Grande y Nuñez-Cacho, 2012 p.78)

Por último, en el estudio de Guillaume, Jones y Wood (2015) se trata de encontrar una manera científica de medir la efectividad del coaching en ámbitos laborales. Para ello, plantean medir el efecto del coaching en tres dimensiones de la actividad laboral: aprendizaje, entrenamiento y desarrollo. En cada uno de estos aspectos, los investigadores medirían los resultados en cuatro dimensiones inherentes al proceso de coaching: retroalimentación, formato de entrega del coaching, coaching interno versus el coaching externo y la duración de coaching. El estudio evidenció que un coaching efectivo se basa en dar una retroalimentación efectiva, con un coach que conozca la cultura de la empresa y más aún si es que proviene de la misma área donde se necesita dar el coach.

Luego del repaso a la literatura existente podemos llegar a las siguientes conclusiones: aún no se ha realizado investigaciones que intenten encontrar la 11 
percepción real que tienen los trabajadores peruanos de la generación "Y" sobre la figura del tutor en ambientes laborales. Lo que sí encontramos son estudios, tanto en el exterior como en el ámbito nacional, en donde se concluye, de manera indirecta, que habría una buena percepción hacia la figura del tutor en el trabajo. Por último, la literatura revisada nos muestra que, a priori, las características de los trabajadores de la generación "Y" son propicias para la introducción de la tutoría y orientación en ambientes laborales. Esto se hace evidente en la necesidad de retroalimentación constante, la cual se puede apoyar en la figura de un mentor que los guie y les enseñe en sus respectivas carreras laborales.

Desde la perspectiva de la teoría revisada y de acuerdo a nuestras necesidades de investigación, se presenta el siguiente objetivo general para el estudio del artículo: descubrir cuál es la imagen que tienen los trabajadores peruanos de la generación "Y" respecto a la figura del tutor en ambientes laborales.

Para alcanzar este objetivo principal de investigación, se plantean los siguientes objetivos secundarios:

a) Evidenciar si existe alguna diferencia significativa, y de qué tipo, en la visión que tienen los trabajadores de la generación "Y" con respecto a la figura del tutor dependiendo del sexo al cual pertenecen.

b) Evidenciar si existe alguna diferencia significativa, y de qué tipo, en la visión que tienen los trabajadores de la generación " $Y$ " respecto a la figura del tutor dependiendo del entorno en dónde trabajan.

c) Evidenciar si existe alguna diferencia significativa, y de qué tipo, en la visión que tienen los trabajadores de la generación "Y" respecto a la figura del tutor dependiendo de si se desempeñan o no en la carrera que han estudiado.

d) Evidenciar si existe alguna diferencia significativa, y de qué tipo, en la visión que tienen los trabajadores de la generación "Y" con respecto a la figura del tutor dependiendo del rubro de la industria donde trabajan.

e) Evidenciar si existe alguna diferencia significativa, y de qué tipo, en la visión que tiene los trabajadores de la generación "Y" con respecto a la figura del tutor dependiendo de la instrucción que posean los trabajadores.

f) Evidenciar si existe alguna diferencia significativa, y de qué tipo, en la visión que tienen los trabajadores de la generación "Y" con respecto a la figura del tutor dependiendo de la cantidad de años que trabajan en una empresa.

\section{Método}

De acuerdo a los fines de la investigación, esta será del tipo descriptiva pues buscará describir las características más resaltantes del objeto de estudio (GómezPeremistré y Reidl, 2010), que en nuestro caso será, las características que los tutores deben tener para los trabajadores peruanos de la generación "Y". Así mismo el diseño será no experimental para la observación del fenómeno y recolección de datos, puesto que el fenómeno no será intervenido y las observaciones se tomarán en el mismo ambiente donde se produce (Gómez-Peremistré y Reidl, 2010), es decir, en los ambientes laborales peruanos de los trabajadores de la generación "Y". Y con respecto al análisis de los datos recolectados, el enfoque será preferentemente cuantitativo. 
Para realizar lo antes mencionado, necesitamos contar con una herramienta de recolección de datos y para ello hemos diseñado un cuestionario ad hoc, la cual no solo servirá para los propósitos de este artículo sino de la investigación doctoral que se realizará más adelante.

El tema de la tutoría y orientación en el mundo laboral tiene un amplio espectro de aplicación y con el fin de acotar la investigación doctoral, se ha decidido introducir un factor de delimitación a las preguntas y es la innovación. La introducción de este factor para ver el efecto de la tutoría está basada en los trabajos revisados en la literatura que han seguido una metodología parecida como son los de Grande y Núñez-Cacho (2012) y Guillaume, Jones y Wood (2015) con el efecto de medir la eficacia del coaching y/o la mentoría. No obstante, para los fines de este artículo, no someteremos a análisis esta dimensión de la encuesta.

Para construir la herramienta de recolección de datos, hemos recurrido a otras encuestas de investigaciones anteriores que apuntan a características laborales de los trabajadores de la generación "Y", a la mentoría en el trabajo y la innovación en el campo laboral:

a) Encuestas Gallup 12 y EFR de Boston para retener talento (Frías, 2014)

b) Encuesta Sistema de Inventario de Transferencia de Aprendizaje (Baron y Morin, 2009), dónde tomaremos los tópicos que nos permitan ver cómo se puede influir en la transferencia de conocimiento en ambientes laborales aplicados a los trabajadores de la generación "Y" para desarrollar la innovación

c) Escala de Orientación al Logro (Kunda, Jordan y Lockwood, 2002) que aparece en el estudio de Chang et. Al (2007), en donde las preguntas nos guiaran para ver como los trabajadores de la generación "Y" pueden alcanzar sus objetivos en la innovación

d) Escala LMX orientada a la relación Coach y Coachee (Graen y Scandura, 1984) que aparece en el estudio de Chang et. Al (2007), con preguntas enfocadas a describir la relación que hay entre el coach y el coachee en ambientes laborales

e) Escala de Satisfacción Laboral (Cook, Wall y Warr, 1979) que aparece en el estudio de Huamán y Vásquez (2012), con la intención de tener una panorámica de cómo se sienten los trabajadores de la generación "Y" con respecto a la relación con los tutores.

f) Cuestionario sobre las funciones del mentoring (Scandura, 2004)

g) Cuestionario Compromiso Organizacional (Allen y Meyer, 1990) que aparece en el trabajo de Frías (2014), para medir el nivel de compromiso con un programa de tutorización de los trabajadores de la generación "Y" y si este se enfoca en desarrollar la Innovación

h) Constructo para medir comportamientos de conocimiento compartido (Brcic y Mihelic, 2015)

i) Encuesta TMX para detectar calidad en las relaciones de los trabajadores de un mismo equipo (Seers, 1989)

j) Modelo de encuesta para encontrar un índice de innovación (Camio et. Al, 2010)

k) Listado de capacidades para la innovación (Bin Ali y Edison, 2010) que aparece en la investigación de Camio et. Al (2010).

De este conjunto de herramientas enumeradas se han tomado y adaptado varias preguntas para el diseño de los diferentes ítems o preguntas de nuestra encuesta ad hoc, las cuales medirán las dimensiones de Innovación, Tutoría y Orientación y 
Características de la Generación "Y". La encuesta contempla estas tres dimensiones pues la misma no solamente ha sido diseñada para la investigación de este artículo sino también para una futura investigación doctoral donde se estudiarán estas tres dimensiones. Y tal como indicamos anteriormente, solamente someteremos a análisis la dimensión de la Tutoría y Orientación a fines de este artículo. Los ítems o preguntas resultantes de esta adaptación se muestran en el Anexo 1.

La encuesta resultante fue sometida a un juicio de expertos con el propósito de conocer si dicho instrumento en verdad mide las variables que pretendemos medir, a través de un análisis de validez de contenido. Para ello, hemos recurrido a la metodología de los juicios de expertos, por lo que pedimos la colaboración de 8 jueces, los cuales provienen del ámbito de la gestión de los recursos humanos en la empresa y además de ser gerentes o jefes de estas áreas.

Los criterios que medirían los jueces en cada pregunta o ítem de la herramienta son:

a) Claridad: el ítem se comprende fácilmente, es decir, su sintaxis y semántica son adecuadas.

b) Coherencia: el ítem tiene relación lógica con la dimensión o indicador que está midiendo.

c) Relevancia: el ítem es esencial o importante, es decir debe ser incluido.

Dichos criterios son por lo general de uso común en distintas pruebas de validez de contenido con respecto a la evaluación de ítems (Badia, 2012).

Para evaluar estos criterios se usó una escala Likert del 1 al 4 donde 1 era deficiente, 2 era aceptable, 3 bueno y 4 se tomó como excelente.

El juicio de los expertos se realizó entre los meses de enero y febrero del año 2018 y tuvo un nivel de respuesta del 75\%. Los resultados obtenidos de los jueces por cada criterio a cada pregunta, fueron sometidos al estadístico W de Kendall el cual nos sirvió para hallar el índice de Concordancia de los jueces al evaluar cada uno de los bloques del cuestionario. De acuerdo a la teoría, una W de Kendall (W) igual a 1 indica una concordancia total entre los jueces que evalúan la herramienta y 0 todo lo contrario.

Otro tema a evaluar es determinar si las concordancias entre los jueces están asociadas ya sea por efectos del azar o las probabilidades o por un acuerdo derivado del proceso del Juicio de Expertos. Para ello usaremos el nivel de significancia (Sig.) que nos permite contrastar la siguiente Hipótesis Nula: Las concordancias entre los jueces se debe a las probabilidades o el azar. En términos de valores, si Sig. es mayor de 0.05 entonces la Hipótesis Nula es válida, contrario sensu la Hipótesis Nula será descartada. Esta estructura y valor del nivel de significancia es sugerida por la mayoría de investigaciones sobre validación de herramientas de medición ya sean existentes o creadas ad hoc en donde un riesgo de concluir que las clasificaciones están asociadas, cuando no lo están, es del 5\% (Badia, 2012)

A continuación, se presenta el cuadro resumen donde se muestra los valores obtenidos sobre la Concordancia de los jueces respecto a las valoraciones de cada bloque de preguntas y luego por la encuesta en general: 
Tabla 1

Índice de Concordancia entre los jueces según W de Kendall (W) y Grado de Significancia (Sig)

\begin{tabular}{|c|c|c|c|c|c|c|}
\hline \multirow[t]{2}{*}{ Valoración } & \multicolumn{2}{|c|}{ Innovación } & \multicolumn{2}{|c|}{ Tutoría y Orientación } & \multicolumn{2}{|c|}{$\begin{array}{l}\text { Características de } \\
\text { trabajadores de la } \\
\text { Generación "Y" }\end{array}$} \\
\hline & W & Sig. & $\mathrm{W}$ & Sig. & $\mathrm{W}$ & Sig. \\
\hline \multirow[t]{2}{*}{$\begin{array}{c}\text { Por bloque de } \\
\text { Preguntas }\end{array}$} & 0.798 & 0.008 & 0.121 & 0.483 & 0.12 & 0.486 \\
\hline & $\mathrm{W}$ & Sig. & & & & \\
\hline General & 0.572 & 0.032 & & & & \\
\hline
\end{tabular}

Nota: Elaboración propia donde W es índice de Kendall y Sig. es Nivel de Significancia.

Con estos datos encontramos que la encuesta tiene un nivel de significancia entre los jueces (sig) de 0.032 y un W de Kendall de 0.572 para la concordancia por lo que podemos concluir que; la encuesta genera un nivel de concordancia adecuado entre los jueces y que además la concordancia no se debe a un efecto de las probabilidades o el azar y que más bien están asociadas entre sí por el mismo proceso. Con estos resultados podemos tomar como validada la encuesta en su estructura interna y que medirá tres factores: Innovación, Tutoría y Orientación laboral y Características de los trabajadores de la generación "Y". Cada uno de estos factores tendrá un grupo de preguntas compuestos por 14 ítems para la innovación, 14 ítems para la tutoría y orientación laboral y 18 ítems para las características de los trabajadores de la generación "Y". Así mismo, cada ítem será evaluado de acuerdo a una escala de Likert del 1 al 5, donde:

-1: Totalmente desacuerdo

-2: En desacuerdo

-3 : Ni de acuerdo ni en desacuerdo

-4: De acuerdo

-5: Totalmente de acuerdo

El detalle de cada ítem y su codificación se presentan en el Anexo 2.

Pasando a la etapa de aplicación de la encuesta, ésta se realizó a un total de 149 participantes de distintos rubros empresariales peruanos entre los meses de junio a octubre de 2018. La determinación del tamaño de la muestra se ha basado en un muestro no probabilístico por conveniencia, pues "permite seleccionar aquellos casos accesibles que acepten ser incluidos. Esto fundamentado en la conveniente accesibilidad y proximidad de los sujetos para el investigador" (Manterola y Otzen, 2017 p. 230)

También es importante tener en cuenta que el número de encuestados estuvo limitado tanto por los recursos materiales como de tiempo con los que contó la investigación. 
Entre los principales datos sociodemográficos laborales de los participantes en la encuesta tenemos:

Tabla 2

Principales datos sociodemográficos de los trabajadores de la generación " $Y$ " peruanos participantes

\begin{tabular}{llcc}
\hline Variables & Categorías & Cantidad & \multicolumn{2}{c}{ Porcentaje } \\
\hline Sexo & Mujer & 70 & $51 \%$ \\
& Hombre & 66 & $49 \%$ \\
\hline Rubro & Servicio & 89 & $65 \%$ \\
& Comercio & 36 & $26 \%$
\end{tabular}

\begin{tabular}{llrr} 
& Manufactura & 11 & $8 \%$ \\
\hline Instrucción & Primaria & 0 & $0 \%$
\end{tabular}

\begin{tabular}{|c|c|c|c|}
\hline & Secundaria & 6 & $4 \%$ \\
\hline & Técnica & 69 & $51 \%$ \\
\hline & Universitaria & 52 & $38 \%$ \\
\hline & Posgrado & 9 & $7 \%$ \\
\hline & Doctorado & 0 & $0 \%$ \\
\hline Entorno & Rural & 12 & $9 \%$ \\
\hline & Urbano & 124 & $91 \%$ \\
\hline Desempeño & $\mathrm{Si}$ & 56 & $41 \%$ \\
\hline & No & 80 & $59 \%$ \\
\hline Tiempo en la empresa & Menos de 6 meses & 37 & $27 \%$ \\
\hline & Entre 6 meses y 1 año & 26 & $19 \%$ \\
\hline & Entre 1 año y 3 años & 40 & $29 \%$ \\
\hline & Más de 3 años & 33 & $24 \%$ \\
\hline
\end{tabular}

Nota: Elaboración propia. La Variable Desempeño debe interpretarse como "Si se desempeña laboralmente en la profesión que estudió". Se consignan los datos de 136 participantes puesto que 13 personas no completaron la encuesta de manera correcta.

El siguiente paso será someter las respuestas de los participantes a los análisis estadísticos respectivos con el fin de obtener las respuestas a nuestros objetivos de investigación. Para Gómez-Peresmitré y Reidl (2010) y Sánchez (2015) existen un grupo de pruebas para este tipo de estudio dónde se presume la existencia de dos o más muestras independientes y estas son las pruebas paramétricas, las cuales se basan en dos supuestos: que el tamaño de muestra sea mayor a 30 individuos y que la distribución de datos es del tipo normal. En este grupo encontramos dos tipos de análisis estadísticos que serán usados de acuerdo a las siguientes necesidades de investigación:

16 
a) $\mathrm{T}$ de Student, que nos permitirá relacionar aquellas variables independientes dicotómicas con las variables dependientes de nuestra encuesta. En el grupo de las variables dicotómicas tenemos: Sexo, Entorno y Desempeño, las cuales se relacionan con los objetivos de investigación a), b) y c) respectivamente. Se asume una hipótesis nula donde se encuentran diferencias significativas entre las variables dependientes y el sexo, desempeño y entorno; respectivamente.

b) ANOVA. Nos permitirá relacionar aquellas variables independientes con más de dos opciones con las variables dependientes de nuestra encuesta. En este grupo tenemos las variables: Rubro, Grado de Instrucción y Tiempo en la empresa, las cuales se relacionan con los objetivos de investigación d), e) y f) respectivamente. Se asume una hipótesis nula donde se encuentran diferencias significativas entre las variables dependientes y el rubro, grado de instrucción y tiempo en la empresa respectivamente.

Para ambos análisis de comparación de medias, se ha decidido tomar un nivel de confianza del $95 \%$ y de significancia del 0.05 , pues son los valores más utilizados en el tipo de investigaciones que realizamos (Gómez-Peresmitré y Reidl, 2010).

La encuesta se realizó a un total de 149 trabajadores de la generación "Y" de distintos rubros empresariales. De este total, 13 encuestas fueron descartadas por las siguientes razones:

- Fuera del rango de edad objetivo

- No llenaron algunos de los datos sociodemográficos laborales

- No llenaron una o más preguntas de la encuesta

Por lo tanto, quedaron un total de 136 encuestas válidas, con lo que el nivel de respuesta fue de $91.3 \%$.

\section{Resultados}

Como vimos anteriormente, tenemos un total de seis objetivos secundarios de investigación que tienen que ver con la figura del tutor, los cuales serán medidos mediante la dimensión Tutoría y Orientación de la encuesta. De estos seis objetivos, los tres primeros se relacionan con encontrar diferencias significativas entre la visión que tienen los trabajadores de la generación " $Y$ " respecto a la figura del tutor dependiendo de las variables Sexo, Entorno y Desempeño. Para poder responder estos objetivos, hemos realizado un análisis T-Student para variables del tipo dicotómicas, como las que analizamos, teniendo los siguientes resultados: 
Tabla 3

Principales estadísticos de la prueba T-Student para el análisis de las variables Sexo, Entorno y Desempeño

\begin{tabular}{|c|c|c|c|c|c|c|c|c|c|c|c|c|}
\hline Ítems & Dimensión & $\begin{array}{c}\text { Categoría } \\
\text { de la } \\
\text { dimensión }\end{array}$ & Medias & $\begin{array}{c}\text { Nivel de } \\
\text { Significancia }\end{array}$ & Dimensión & $\begin{array}{c}\text { Categoría } \\
\text { de la } \\
\text { dimensión }\end{array}$ & Medias & $\begin{array}{c}\text { Nivel de } \\
\text { Significancia }\end{array}$ & Dimensión & $\begin{array}{c}\text { Categoría } \\
\text { de la } \\
\text { dimensión }\end{array}$ & Medias & $\begin{array}{c}\text { Nivel de } \\
\text { Significancia }\end{array}$ \\
\hline \multirow{2}{*}{ OYT1 } & \multirow{2}{*}{ SEXO } & Mujer & 3,67 & \multirow{2}{*}{, 829} & \multirow{2}{*}{ ENTORNO } & Rural & 3,67 & \multirow{2}{*}{,922 } & \multirow{2}{*}{ DESEMPEÑO } & $\mathrm{Si}$ & 3,77 & \multirow{2}{*}{,483 } \\
\hline & & Hombre & 3,71 & & & Urbano & 3,70 & & & No & 3,63 & \\
\hline \multirow{2}{*}{ OYT2 } & \multirow{2}{*}{ SEXO } & Mujer & 3,61 & \multirow{2}{*}{,689 } & \multirow{2}{*}{ ENTORNO } & Rural & 3,58 & \multirow{2}{*}{,801 } & \multirow{2}{*}{ DESEMPEÑO } & $\mathrm{Si}$ & 3,57 & \multirow{2}{*}{,469 } \\
\hline & & Hombre & 3,68 & & & Urbano & 3,66 & & & No & 3,70 & \\
\hline \multirow{2}{*}{ OYT3 } & \multirow{2}{*}{ SEXO } & Mujer & 3,67 & \multirow{2}{*}{, 267} & \multirow{2}{*}{ ENTORNO } & Rural & 3,25 & \multirow{2}{*}{, 065} & \multirow{2}{*}{ DESEMPEÑO } & $\mathrm{Si}$ & 3,88 & \multirow{2}{*}{,279 } \\
\hline & & Hombre & 3,86 & & & Urbano & 3,81 & & & No & 3,68 & \\
\hline \multirow{2}{*}{ OYT4 } & \multirow{2}{*}{ SEXO } & Mujer & 3,84 & \multirow{2}{*}{, 534} & \multirow{2}{*}{ ENTORNO } & Rural & 3,83 & \multirow{2}{*}{, 824} & \multirow{2}{*}{ DESEMPEÑO } & $\mathrm{Si}$ & 3,95 & \multirow{2}{*}{, 481} \\
\hline & & Hombre & 3,94 & & & Urbano & 3,89 & & & No & 3,84 & \\
\hline & & Mujer & 3,70 & & & Rural & 3,33 & & & $\mathrm{Si}$ & 3,80 & \\
\hline OYT5 & SEXO & Hombre & 3,73 & ,876 & ENTORNO & Urbano & 3,75 & , 177 & DESEMPENO & No & 3,65 &, 374 \\
\hline & & Mujer & 3,93 & & & Rural & 4,08 & & & $\mathrm{Si}$ & 4,05 & \\
\hline OYT6 & SEXO & Hombre & 3,94 & ,945 & ENTORNO & Urbano & 3,92 & 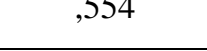 & DESEMPENO & No & 3,84 &, 171 \\
\hline & & Mujer & 3,91 & & & Rural & 3,92 & & & $\mathrm{Si}$ & 3,98 & \\
\hline OYT7 & SEXO & Hombre & 3,94 &, 874 & ENTORNO & Urbano & 3,92 & ,994 & DESEMPENO & No & 3,87 & ,498 \\
\hline OYT8 & SFXO & Mujer & 3,74 & 708 & FNTORNO & Rural & 3,67 & 714 & DFSFMPEÑ & $\mathrm{Si}$ & 3,66 & 330 \\
\hline $0 Y 18$ & SEXU & Hombre & 3,79 & , 198 & ENIURINU & Urbano & 3,78 &,$/ 14$ & DESEMIPENO & No & 3,84 &, 330 \\
\hline ОYTQ & & Mujer & 3,63 & & & Rural & 3,50 & & & $\mathrm{Si}$ & 3,77 & \\
\hline ОYТ9 & SEXO & Hombre & 3,73 & , 550 & ENTORNO & Urbano & 3,69 & ,513 & DESEMPENO & No & 3,61 &, 341 \\
\hline & & Mujer & 3,69 & & & Rural & 3,75 & & & $\mathrm{Si}$ & 3,88 & \\
\hline ОYТ10 & SEXO & Hombre & 3,88 & ,244 & ENTORNO & Urbano & 3,78 & ,917 & DESEMPENO & No & 3,71 &, 327 \\
\hline & & Mujer & 3,83 & & & Rural & 3,83 & & & $\mathrm{Si}$ & 3,93 & \\
\hline OYТ11 & SEXO & Hombre & 3,86 & , 815 & ENTORNO & Urbano & 3,85 & ,963 & DESEMPENO & No & 3,78 &, 347 \\
\hline & SEYO & Mujer & 3,79 & 860 & & Rural & 3,25 & 057 & DFSEMPFÑ & $\mathrm{Si}$ & 3,91 & 100 \\
\hline $0 Y 112$ & SEXU & Hombre & 3,76 & ,869 & EINTURINO & Urbano & 3,82 & ו & DESEIMIPENO & No & 3,68 & , 190 \\
\hline OYT13 & SEXO & Mujer & 3,86 & 338 & FNTORNO & Rural & 3,67 & 281 & DFSFMPF ÑO & $\mathrm{Si}$ & 4,07 & 102 \\
\hline 01115 & SEAU & Hombre & 4,00 & ,538 & ENIUKINU & Urbano & 3,95 & ,281 & DESEIVIPENO & No & 3,82 &, 102 \\
\hline OYT14 & SEXO & Mujer & 3,74 & 865 & ENTORNO & Rural & 3,83 & 784 & DESFMPFÑO & $\mathrm{Si}$ & 3,79 & 720 \\
\hline & SEXU & Hombre & 3,77 & ,865 & EINTURINO & Urbano & 3,75 &,$/ 84$ & DESEIMIPENO & No & 3,72 &,$/ 20$ \\
\hline
\end{tabular}

Nota: Elaboración propia.

18

(2019) MLSER, 3(2), 7-32 
De la tabla se deduce que ningún valor de significancia para la dimensión Orientación y Tutoría con respecto a las variables sexo, entorno y desempeño es menor a 0.05 por lo que nos lleva a rechazar al hipótesis nula que da por sentada que existen diferencias significativas, por lo que se poder concluir que no se evidencian diferencias significativas con respecto a la visión de la figura del tutor por parte de los trabajadores de la generación "Y" de acuerdo a las variables Sexo, Entorno ni Desempeño.

Continuando con las respuestas a nuestros objetivos de investigación, el siguiente grupo a analizar son aquellos que se relacionan con encontrar diferencias significativas entre la visión que tienen los trabajadores de la generación "Y" respecto a la figura del tutor dependiendo de las variables Instrucción y Tiempo de permanencia en la empresa. Para poder responder estos objetivos, hemos realizado un análisis ANOVA para variables que tienen más de dos posibles respuestas, como las que analizamos, teniendo los siguientes resultados:

Tabla 4

Principales estadísticos de la prueba ANOVA para el análisis de las variables Instrucción Tiempo de permanencia en la empresa y Rubro de la empresa

\begin{tabular}{|c|c|c|c|c|c|}
\hline $\begin{array}{c}\text { Ítem/Variable } \\
\text { dependiente }\end{array}$ & $\begin{array}{r}\text { Categc } \\
\text { inde }\end{array}$ & $\begin{array}{l}\text { ría variable } \\
\text { pendiente }\end{array}$ & $\begin{array}{c}\text { Diferencia } \\
\text { de medias (I- } \\
\mathrm{J} \text { ) }\end{array}$ & $\begin{array}{l}\text { Desv. } \\
\text { Error }\end{array}$ & Sig. \\
\hline \multirow[t]{2}{*}{ OYT1 } & Universitaria & Secundaria &, $962^{*}$ & 0.464 & 0.040 \\
\hline & & Técnica &, $425^{*}$ & 0.197 & 0.033 \\
\hline OYT2 & Universitaria & Técnica &, $392^{*}$ & 0.178 & 0.029 \\
\hline OYT6 & Universitaria & Técnica &, $535^{*}$ & 0.163 & 0.001 \\
\hline OYT8 & Universitaria & Técnica &, $454^{*}$ & 0.185 & 0.015 \\
\hline OYT9 & Universitaria & Técnica &, $348^{*}$ & 0.175 & 0.049 \\
\hline OYT10 & Universitaria & Técnica &, $425^{*}$ & 0.175 & 0.016 \\
\hline OYT11 & Universitaria & Técnica &, $415^{*}$ & 0.157 & 0.009 \\
\hline \multirow[t]{2}{*}{ OYT12 } & Universitaria & Secundaria & ,840* & 0.407 & 0.041 \\
\hline & & Técnica & ,666" & 0.173 & 0.000 \\
\hline OYT13 & Universitaria & Técnica & ,588 & 0.152 & 0.000 \\
\hline OYT14 & Universitaria & Técnica & $478^{*}$ & 0.184 & 0.011 \\
\hline OYT4 & $\begin{array}{l}\text { Menos de } 6 \\
\text { meses }\end{array}$ & $\begin{array}{l}\text { Entre } 6 \text { meses y } \\
1 \text { año }\end{array}$ &, $454^{*}$ & 0.229 & 0.050 \\
\hline \multirow[t]{2}{*}{ OYT7 } & $\begin{array}{l}\text { Menos de } 6 \\
\text { meses }\end{array}$ & $\begin{array}{l}\text { Entre } 1 \text { año y } 3 \\
\text { años }\end{array}$ & $418^{*}$ & 0.206 & 0.045 \\
\hline & & Más de 3 años &, $455^{*}$ & 0.217 & 0.038 \\
\hline
\end{tabular}

Nota: Elaboracion propia. * Diferencias de medias es significativas en el nivel de 0.05 . Extracto de la tabla completa presentada en el anexo 3 con aquellas variables que presentan diferencias significativas.

Las evidencias que nos muestra la tabla anterior se pueden resumir en dos grandes rubros: evidencias con respecto a la variable independiente instrucción y evidencias con respecto a la variable independiente tiempo de permanencia en la empresa. Hecha esta primera división pasaremos a presentar las evidencias específicas en cada grupo. 
En las evidencias que tienen relación con la variable independiente instrucción se evidencian diferencias significativas entre aquellos trabajadores que tienen instrucción universitaria con respecto a aquellos que tienen instrucción técnica en los ítems que tienen que ver con la orientación y tutoría:

-OYT1: Recibo retroalimentación sobre mi trabajo de parte de mi jefe

-OYT2: Mi jefe es receptivo a la retroalimentación que yo le puedo hacer

-OYT6: Mi relación con mi jefe es positiva

-OYT8: Mi jefe conoce las dificultades y problemas del área y realiza acciones para resolverlas

-OYT9: Mi jefe ha sido un factor importante para integrarme o desenvolverme adecuadamente dentro de mi trabajo

-OYT10: Mi jefe me genera confianza

-OYT11: Las sesiones de retroalimentación con mi jefe o tutor me permiten reflexionar y proyectar metas a futuro en mi trabajo

-OYT12: Mi tutor o jefe apoya mis acciones y/o decisiones

-OYT13: Mi jefe me apoya cuando debo resolver un problema en el trabajo

-OYT14: Reconozco a mi jefe como una persona que me motiva

Siendo que en la mayoría de casos se evidencia una tendencia más favorable a todas estas prácticas entre los trabajadores con instrucción universitaria. Casos especiales podemos encontrar en los ítems: recibo retroalimentación sobre mi trabajo de parte de mi jefe (OYT1) y mi tutor o jefe apoya mis acciones y/o decisiones (OYT12); en donde las evidencias de diferencias significativas se extienden al grupo de trabajadores con instrucción secundaria.

El siguiente grupo refiere a la variable independiente de tiempo de permanencia en la empresa y en ella las evidencias de diferencias significativas se dan para la dimensión orientación y tutoría, en los ítems mi jefe es honesto conmigo (OYT4) y mi jefe tiene el conocimiento necesario para orientarme en mis actividades (OYT7) se evidencia que existen diferencias significativas a favor en aquellos trabajadores que tienen menos de 6 meses con respecto a aquellos que tienen más tiempo.

En el último grupo, con respecto a la variable Rubro de la empresa, no se evidencia ningún dato que demuestre que existe una diferencia significativa, por lo cual no se muestran en la tabla 4 resultados al respecto.

\section{Discusión y conclusiones}

Lo primero que nos arroja esta investigación es que entre las variables independientes sexo, entorno, desempeño y rubro de la empresa y la dimensión orientación y tutoría, no se hallan diferencias significativas por lo que estas variables no influyen en la visión que tienen los trabajadores peruanos de la generación "Y" sobre la figura del tutor en ambientes laborales.

Por otro lado, el análisis de las variables instrucción y tiempo de permanencia en la empresa para con la dimensión Orientación y Tutoría aportaron mucha más información para explicar la figura que tiene el trabajador de la generación "Y" con respecto a la figura del tutor en el mundo laboral. En especial se puede ver que la variable instrucción del trabajador tiene relaciones significativas con la visión de la figura del tutor en la empresa y siendo más específicos, con los trabajadores de la generación "Y" que tienen una educación universitaria, que están a favor de tener un tutor en su labor diaria. 
Con los resultados propuestos podemos concluir que existe una imagen que tienen los trabajadores peruanos de la generación "Y" con respecto a la figura del tutor en ambientes laborales cuyas características son las siguientes:

a) Es una persona que da retroalimentación sobre el trabajo realizado

b) Es receptivo a la retroalimentación que el trabajador le pueda hacer

c) Que se puede tener una relación positiva con él

d) Que conoce el área, sus problemas y dificultades, pero que además realiza acciones para resolver

e) Que es una persona importante en la integración y posterior desenvolvimiento en el área de sus trabajadores

f) Que genera un clima de confianza

g) Que las retroalimentaciones que da son sustanciosas y permiten reflexionar y a la vez proyectar metas a futuro en su trabajo diario

h) Que apoya las decisiones y acciones de sus trabajadores

i) Es una persona que apoya al trabajador al momento de resolver problemas

j) Y que motiva a realizar las cosas

En estos puntos podemos ver que existe una coincidencia con otros estudios que se han revisado en la bibliografía como son Grande y Nuñez-Cacho (2012) y Dávila y Mita (2015) en dónde apuntan que los trabajadores de la Generación "Y" ven con buenos ojos la figura de un mentor/tutor/jefe ya sea como herramienta de desarrollo profesional y/o como de retención de talento y además con las características de dar retroalimentación, motivar a realizar las cosas y ser un soporte en el desarrollo laboral de estos trabajadores.

Además del modelo del tutor, un dato que no resulta menos relevante es la coincidencia con los estudios de Newell (2015) y Dávila y Mita (2015) en que los principales hallazgos se han realizado en poblaciones laborales con estudios superiores siendo principalmente los de instrucción universitaria donde más hallazgos se han hecho.

A esto podemos sumar que, de acuerdo a la literatura revisada, las características de los trabajadores de la generación "Y" que más compatibilizan con las características de la figura del tutor que hemos encontrado son:

a) El sentir que el trabajo tiene un sentido para ellos

b) Sentir al jefe como una persona que guíe y enseñe

c) Percibir que reciben críticas constructivas sobre su desempeño laboral

Con respecto a las limitaciones de este estudio, la más importantes es la cantidad de personas que participaron. Esta limitación se debió al factor tiempo, pues en el periodo en que se aplicaron las encuestas, la mayoría de empresas estaban también realizando las encuestas anuales de clima laboral, por lo que muchas se negaron a saturar a sus trabajadores con encuestas adicionales.

Dentro de las cuestiones que quedarían por estudiar están el comprobar si los resultados sobre la figura del tutor en ambientes laborales para trabajadores que no tienen educación superior coinciden o difieren con respecto a los trabajadores con instrucción superior.Así mismo, quedaría por investigar toda la dimensión de la Innovación de la encuesta con respecto a la figura del tutor, tema que se estudiará en la investigación doctoral posterior.

Por último, con la cantidad de trabajadores encuestados de la generación "Y" se ha observado que las variables sexo, entorno, desempeño y rubro no presenta diferencias significativas con respecto a la visión que tienen los mismos sobre la figura 
del tutor. Esto se debe comprobar si se mantiene al aumentar la cantidad de trabajadores encuestados.

\section{Referencias}

Badia, D. (2012). Metodología de los mapas de concordancia para la estratificación de variables cuantitativas: Aplicación a la asignatura de Medidas Electrónicas. Tesis doctoral sin publicar. Universidad Ramon Llull, Cataluña.

Baron, L. \& Morin, L. (2009). The Coach-Coachee Relantioship in Executive Coaching: A Field Study. [Versión electronica]. Human Resource Development Quarterly 20 (1), 85-106 Doi: 10.1002/hrdq.20009

Begazo, J. y Fernandez, W. (2015). Los Millennials peruanos: Características y Proyecciones de Vida. Gestión en el Tercer Milenio. Revista de Investigación de la Facultad de Ciencias Administrativas Universidad Nacional Mayor de San Marcos, 18(36), 9- 15. $\quad$ Retrieved from http://revistasinvestigacion.unmsm.edu.pe/index.php/administrativas/article/vie $\underline{\mathrm{w} / 11699 / 10489}$

Brcic, Z. y Mihelic, K. (2015). Knowledge sharing between different generations of employees: an example from Slovenia. Economic Research-Ekonomska Istrazivanja 28(1), 853-867. doi: 10.1080/1331677X.2015.1092308

Boschma, J. y Groen, I. (2008). Generación Einstein. Más listos, más rápidos y más sociables. Madrid:Ediciones Gestión 2000

Camio, M., Rébori, A. y Romero, M. (2010). Un Índice para "medir" el nivel de innovación tecnológica en empresas intensivas en el uso de tecnología. [Versión elec-trónica] Revista de Administración e Innovación 7(1), 3-20.

Cannice, M., Koontz, H. y Weihrich, H. (2012). Administración: una perspectiva global y empresarial (14 ${ }^{\mathrm{a}}$ ed.) Mexico: McGraw-Hill Interamericana

Carrasco, E. (2014). De la capacitación al desarrollo organizacional y de éste hacia la ambidestreza. novaRua revista universitaria de administración Universidad Autónoma de Ciudad Juárez, 4(9), 25-33

Castro, J. y Yamada, G. (2010). La economía peruana en la última década: Mejor desempeño y mayor resilencia a la crisis. Trabajo presentado en la Conferencia Internacional Políticas Económicas en América Latina Quito: Servicios de publicaciones de la Pontificia Universidad Católica del Ecuador.

Coll, C., Mauri, T. y Onrubia, J. (2008). Ayudar a aprender en contextos educativos: el ejercicio de la influencia educativa y el análisis de la enseñanza. Revista de Educación, 346, 33-70

Chang, Y., Jeng Lin, W. \& Yu Lin, Ch. (2017). The impact of coaching orientation on subordinate performance: the moderating effects of implicit person theory and LMX. Asia Pacific Journal of Human Resources, 55, 86-10

Dávila, C. y Mitta, D. (2015). Retención del talento humano: Políticas y prácticas aplicadas sobre jóvenes "Millennials" en grupo de organizaciones. Tesis presentada para obtener el título profesional de Licenciado en Gestión, con mención en Gestión Empresarial, Facultad de Gestión y Alta Dirección, Pontificia Universidad 
Católica del Perú, Lima. Retrieved from http://tesis.pucp.edu.pe/repositorio/handle/123456789/6948

Deloitte (2015). Brechas Importantes Encuesta Deloitte 2015 Generación del Milenio. Retrieved from http://www2.deloitte.com/content/dam/Deloitte/cr/Documents/humancapital/estudios/150225-EncuestaDeloitte2015-Generacion-del-Milenio.pdf

Epstein, M. \& Hershatter, A. (2006). Is yout Firm ready for the Millennials?. Retrieved from http://knowledge.emory.edu/article.cfm?articleid=950\#

Espinosa, F. (2011). Diseño y Construcción de Organizaciones Inteligentes. Recuperado de http://www.otr.com.mx/compartidos/Org\%20Int.pdf

Frías, P. (2014). Compromiso y satisfacción laboral como factores de permanencia de la generación $Y$. Tesis de magister sin publicar, Universidad de Chile, Santiago de Chile.

Gonzáles, R. (2011). La incorporación de la Generación "Y" al mercado laboral. El caso de una entidad financiera de la ciudad de Resistencia. Palermo Business Review $567-93$

Grande, F. y Nuñez-Cacho, P. (2012). El desarrollo de los recursos humanos a través del mentoring: El caso español. [Versión electrónica] Intangible Capital, 8(1), 61-91.

Great Place to Work (2013). Generación Y: ¿Cómo retener a la generación que siempre se quiere ir?. Retrieved from http://www.greatplacetowork.com.pe/storage/documents/GPTWP2 _2014_AGO__Reporte_GPTW_-_Generacion_Y_-_V2.pdf

Gómez-Peresmitré, G. y Reidl, L. (2010). Metodología de Investigación en Ciencias Sociales. México: U.N.A.M

Guillaume, Y., Jones, R. \& Woods, S. (2015). The effectiveness of workplace coaching: A meta-analysis of learning and performance outcomes from coaching. Journal of Occupational and Organizational Psychology 89(2), 249-277 doi: 10.1111/joop.12119

Howe, N. \& Strauss, W. (2000). Millennials Rising: The Next Great Generation. London. Vintage Books

Huamán, I. y Vásquez, M. (2012). Habilidades sociales y tutoría docente según la percepción de los alumnos de 6to grado: Red Educativa Ventanilla. Tesis de magister sin publicar, Universidad San Ignacio de Loyola, Lima.

Manterola, C. y Otzen, T. (2017). Técnicas de Muestreo sobre una Población a Estudio. Int. J. Morphol, 35(1), 227-232.

Newell, T. (2015). Propuesta de formación Social Learning 2.0 para la generación " $Y$ ". Tesis para optar el grado académico de Master en Dirección Estratégica del Fac-tor Humano, Programa de Master en Dirección Estratégica del Factor Humano, Universidad Peruana de Ciencias Aplicadas, Lima. Retrieved from http://hdl.handle.net/10757/576181

Penagos, T. y Rubio, E (2014). Millennials y Millennials peruanos. Realidad, expectativas y proyecciones. Lima: ER|Roland 
Pricewaterhouse Coopers (2014). ler estudio: Tendencias de Retención del talento Perú 2014. Un desafío para las organizaciones de hoy. Lima: Autor

Sanchez, R. (2015). T-Student: Usos y Abusos. Revista Mexicana de Cardiología, 26(1), 59-61.

Retrieved

from

http://www.scielo.org.mx/scielo.php?script=sci_arttext\&pid=S0188-

$\underline{21982015000100009}$

Serrat, O. (2010). Coaching and mentoring. Washington, DC: Asian Development Bank. Retrieved from http://digitalcommons.ilr.cornell.edu/intl/114

Schalk, E. (2005). Modelo de enseñanza-aprendizaje para adultos en la Era del Conocimiento. Diseño y estructura del Modelo. Gestión en el Tercer Milenio, Revista de la facultad de Ciencias Administrativas UNMSM, 8 (16), 63-87.

Fecha de recepción: 09/01/2019

Fecha de revisión: 10/01/2019

Fecha de aceptación: 10/06/2019 
Anexo 1

Anexos

Ítems de la encuesta antes de la validación por los jueces

\begin{tabular}{|c|c|}
\hline $\begin{array}{l}\text { Codificación } \\
\text { de los ítems }\end{array}$ & Redacción de los ítems \\
\hline INN1 & En el tiempo que trabaja en la empresa, ha visto nuevas formas de trabajo, servicio y/o producto. \\
\hline INN2 & La empresa recurre a sus trabajadores para tomar ideas innovadoras \\
\hline INN3 & En mi actividad diaria, puedo volcar los conocimientos nuevos que voy adquiriendo \\
\hline INN4 & En la empresa hay tolerancia al error \\
\hline INN5 & La empresa recompensa la generación de nuevas ideas \\
\hline INN6 & Existe una actitud de apertura al cambio en la empresa \\
\hline INN7 & Me siento motivado a aprender algo nuevo \\
\hline INN8 & Me enfrento, a menudo, con situaciones nuevas e inusuales en mi labor diaria \\
\hline INN9 & Prefiero los enfoques únicos y originales a cada proyecto que se me encomienda \\
\hline INN10 & Suelo actuar anticipándome a futuros problemas, necesidades o cambios en mi trabajo \\
\hline INN11 & Estoy a favor de la experimentación y aproximaciones originales al resolver problemas laborales \\
\hline INN12 & Siento que puedo compartir con mis pares información \\
\hline INN13 & Siento que la compañía tiene procesos y/o productos más innovadores que la competencia \\
\hline INN14 & Tengo curiosidad por investigar y saber más sobre el negocio de la empresa \\
\hline INN15 & Puedo cambiar la forma de trabajar si es que cambian las condiciones de mi entorno \\
\hline INN16 & Cuestiono, constructivamente, la forma de hacer las cosas \\
\hline INN17 & Utilizo las herramientas digitales para hacer mis labores diarias más ágiles y eficientes \\
\hline INN18 & Busco oportunidades para aportar nuevas ideas en mis labores diarias \\
\hline INN19 & Soy receptivo a las nuevas ideas de mis pares en el trabajo \\
\hline INN20 & Genero más de una alternativa de solución a los problemas en mi ambiente de trabajo \\
\hline INN21 & Genero una lluvia de ideas en la búsqueda de soluciones a problemas complejos \\
\hline OYT1 & Recibo retroalimentación sobre mi trabajo de parte de mi jefe \\
\hline OYT2 & Mi jefe es receptivo a la retroalimentación que yo le puedo hacer \\
\hline OYT3 & Recibo ayuda para aprender y/o crecer profesionalmente \\
\hline OYT4 & Mi jefe es honesto conmigo \\
\hline OYT5 & Siento que se me asesora sobre temas importantes en mi trabajo diario \\
\hline
\end{tabular}

(2019) MLSER, 3(2), 7-32 
OYT6 Mi relación con mi jefe es positiva

OYT7 Mi jefe tiene el conocimiento necesario para orientarme en mis actividades

OYT8 Mi jefe conoce las dificultades y problemas del área y realiza acciones para resolverlas

OYT9 Mi jefe ha sido un factor importante para integrarme o desenvolverme adecuadamente dentro de mi trabajo

OYT10 Mi jefe me genera confianza

OYT11 Las sesiones de retroalimentación con mi jefe o tutor me permiten reflexionar y proyectar metas a futuro en mi trabajo

OYT12 Mi tutor o jefe apoya mis acciones y/o decisiones

OYT13 Mi jefe me apoya cuando debo resolver un problema en el trabajo

OYT14 Reconozco a mi jefe como una persona que me motiva

OYT15 Mi jefe procura que tenga todas las herramientas necesarias para que pueda desarrollar mis labores

ACT1 Tengo claro lo que se espera de mí en la organización

ACT2 Recibo reconocimiento por mi trabajo

ACT3 Se preocupan por mí, como persona, en mi trabajo

ACT4 Siento que puedo desarrollar todo mi potencial

ACT5 Mis opiniones cuentan en el trabajo

ACT6 Me siento comprometido con la misión de la empresa

ACT7 Siento que mi trabajo tiene sentido para mí

ACT8 Se propicia un ambiente de trabajo en equipo en donde laboro

ACT9 Recibo críticas constructivas sobre mi desempeño laboral

ACT10 Siento a mi jefe como una persona que guía y enseña

ACT11 Siento que tengo libertad para elegir mi propio método de trabajo

ACT12 Me siento orgulloso del lugar donde trabajo

ACT13 Siento que podría quedarme mucho tiempo en esta empresa

ACT14 Siento que existe un ambiente de confianza entre mis pares

ACT15 A menudo ofrezco mi ayuda para realizar el trabajo del área o equipo

ACT16 En mi entorno laboral, me siento en la libertad de usar herramientas digitales para mis labores diarias

ACT17 En mi trabajo, el tipo de comunicación con mis pares y jefes es inmediata

ACT18 El ambiente de trabajo donde laboro, propicia el uso de la tecnología 
Anexo 2

Ítems de la encuesta después de la validación de los jueces

\begin{tabular}{|c|c|}
\hline $\begin{array}{l}\text { Codificación } \\
\text { de los ítems }\end{array}$ & Redacción de los ítems \\
\hline INN1 & La empresa recurre a sus trabajadores para tomar ideas innovadoras \\
\hline INN2 & En mi actividad diaria, puedo volcar los conocimientos nuevos que voy adquiriendo \\
\hline INN3 & En la empresa hay tolerancia al error \\
\hline INN4 & La empresa recompensa la generación de nuevas ideas \\
\hline INN5 & Existe una actitud de apertura al cambio en la empresa \\
\hline INN6 & Me siento motivado a aprender algo nuevo \\
\hline INN7 & Prefiero los enfoques únicos y originales a cada proyecto que se me encomienda \\
\hline INN8 & Suelo actuar anticipándome a futuros problemas, necesidades o cambios en mi trabajo \\
\hline INN9 & Siento que puedo compartir con mis pares información \\
\hline INN10 & Siento que la compañía tiene procesos y/o productos más innovadores que la competencia \\
\hline INN11 & Tengo curiosidad por investigar y saber más sobre el negocio de la empresa \\
\hline INN12 & Cuestiono, constructivamente, la forma de hacer las cosas \\
\hline INN13 & Busco oportunidades para aportar nuevas ideas en mis labores diarias \\
\hline INN14 & Genero más de una alternativa de solución a los problemas en mi ambiente de trabajo \\
\hline OYT1 & Recibo retroalimentación sobre mi trabajo de parte de mi jefe \\
\hline OYT2 & Mi jefe es receptivo a la retroalimentación que yo le puedo hacer \\
\hline OYT3 & Recibo ayuda para aprender y/o crecer profesionalmente \\
\hline OYT4 & Mi jefe es honesto conmigo \\
\hline OYT5 & Siento que se me asesora sobre temas importantes en mi trabajo diario \\
\hline OYT6 & Mi relación con mi jefe es positiva \\
\hline OYT7 & Mi jefe tiene el conocimiento necesario para orientarme en mis actividades \\
\hline OYT8 & Mi jefe conoce las dificultades y problemas del área y realiza acciones para resolverlas \\
\hline OYT9 & Mi jefe ha sido un factor importante para integrarme o desenvolverme adecuadamente dentro de mi trabajo \\
\hline OYT10 & Mi jefe me genera confianza \\
\hline OYT11 & Las sesiones de retroalimentación con mi jefe o tutor me permiten reflexionar y proyectar metas a futuro en mi trabajo \\
\hline OYT12 & Mi tutor o jefe apoya mis acciones y/o decisiones \\
\hline OYT13 & Mi jefe me apoya cuando debo resolver un problema en el trabajo \\
\hline OYT14 & Reconozco a mi jefe como una persona que me motiva \\
\hline 27 & \\
\hline
\end{tabular}

(2019) MLSER, 3(2), 7-32 
Tengo claro lo que se espera de mí en la organización

Recibo reconocimiento por mi trabajo

Se preocupan por mí, como persona, en mi trabajo

Siento que puedo desarrollar todo mi potencial

Mis opiniones cuentan en el trabajo

Me siento comprometido con la misión de la empresa

Siento que mi trabajo tiene sentido para mí

Se propicia un ambiente de trabajo en equipo en donde laboro

Recibo críticas constructivas sobre mi desempeño laboral

Siento a mi jefe como una persona que guía y enseña

Siento que tengo libertad para elegir mi propio método de trabajo

Me siento orgulloso del lugar donde trabajo

Siento que podría quedarme mucho tiempo en esta empresa

Siento que existe un ambiente de confianza entre mis pares

A menudo ofrezco mi ayuda para realizar el trabajo del área o equipo

En mi entorno laboral, me siento en la libertad de usar herramientas digitales para mis labores diarias

En mi trabajo, el tipo de comunicación con mis pares y jefes es inmediata

El ambiente de trabajo donde laboro, propicia el uso de la tecnología 


\section{Anexo 3}

Estadísticos de la prueba ANOVA para el análisis de la variable Rubro de la empresa con respecto a la Orientación y Tutoría

\begin{tabular}{|c|c|c|c|c|c|}
\hline Ítem & \multicolumn{2}{|r|}{$\begin{array}{l}\text { Dimensión Tiempo de permanencia } \\
\text { Categoría }\end{array}$} & $\begin{array}{l}\text { Diferencia de medias } \\
\text { (I-J) }\end{array}$ & Desv. Error & \multirow{2}{*}{$\begin{array}{c}\text { Sig. } \\
0.348\end{array}$} \\
\hline \multirow[t]{6}{*}{ OYT1 } & SERVICIO & COMERCIO & -0.204 & 0.217 & \\
\hline & & MANUFACTURA & -0.098 & 0.350 & 0.780 \\
\hline & COMERCIO & SERVICIO & 0.204 & 0.217 & 0.348 \\
\hline & & MANUFACTURA & 0.106 & 0.378 & 0.779 \\
\hline & MANUFACTURA & SERVICIO & 0.098 & 0.350 & 0.780 \\
\hline & & COMERCIO & -0.106 & 0.378 & 0.779 \\
\hline \multirow[t]{6}{*}{ OYT2 } & SERVICIO & COMERCIO & -0.154 & 0.194 & 0.427 \\
\hline & & MANUFACTURA & -0.132 & 0.314 & 0.675 \\
\hline & COMERCIO & SERVICIO & 0.154 & 0.194 & 0.427 \\
\hline & & MANUFACTURA & 0.023 & 0.338 & 0.947 \\
\hline & MANUFACTURA & SERVICIO & 0.132 & 0.314 & 0.675 \\
\hline & & COMERCIO & -0.023 & 0.338 & 0.947 \\
\hline \multirow[t]{6}{*}{ OYT3 } & SERVICIO & COMERCIO & -0.053 & 0.200 & 0.792 \\
\hline & & MANUFACTURA & 0.026 & 0.324 & 0.937 \\
\hline & COMERCIO & SERVICIO & 0.053 & 0.200 & 0.792 \\
\hline & & MANUFACTURA & 0.078 & 0.349 & 0.823 \\
\hline & MANUFACTURA & SERVICIO & -0.026 & 0.324 & 0.937 \\
\hline & & COMERCIO & -0.078 & 0.349 & 0.823 \\
\hline \multirow[t]{6}{*}{ OYT4 } & SERVICIO & COMERCIO & -0.085 & 0.178 & 0.636 \\
\hline & & MANUFACTURA & 0.251 & 0.289 & 0.385 \\
\hline & COMERCIO & SERVICIO & 0.085 & 0.178 & 0.636 \\
\hline & & MANUFACTURA & 0.336 & 0.311 & 0.282 \\
\hline & MANUFACTURA & SERVICIO & -0.251 & 0.289 & 0.385 \\
\hline & & COMERCIO & -0.336 & 0.311 & 0.282 \\
\hline
\end{tabular}

29

(2019) MLSER, 3(2), 7-32 


\begin{tabular}{|c|c|c|c|c|c|}
\hline \multirow[t]{6}{*}{ OYT5 } & \multirow[t]{2}{*}{ SERVICIO } & COMERCIO & -0.008 & 0.200 & 0.966 \\
\hline & & MANUFACTURA & 0.378 & 0.324 & 0.245 \\
\hline & \multirow[t]{2}{*}{ COMERCIO } & SERVICIO & 0.008 & 0.200 & 0.966 \\
\hline & & MANUFACTURA & 0.386 & 0.349 & 0.270 \\
\hline & \multirow[t]{2}{*}{ MANUFACTURA } & SERVICIO & -0.378 & 0.324 & 0.245 \\
\hline & & COMERCIO & -0.386 & 0.349 & 0.270 \\
\hline \multirow[t]{6}{*}{ OYT6 } & \multirow[t]{2}{*}{ SERVICIO } & COMERCIO & 0.089 & 0.181 & 0.625 \\
\hline & & MANUFACTURA & 0.250 & 0.293 & 0.394 \\
\hline & \multirow[t]{2}{*}{ COMERCIO } & SERVICIO & -0.089 & 0.181 & 0.625 \\
\hline & & MANUFACTURA & 0.162 & 0.316 & 0.610 \\
\hline & \multirow[t]{2}{*}{ MANUFACTURA } & SERVICIO & -0.250 & 0.293 & 0.394 \\
\hline & & COMERCIO & -0.162 & 0.316 & 0.610 \\
\hline \multirow[t]{6}{*}{ OYT7 } & \multirow[t]{2}{*}{ SERVICIO } & COMERCIO & 0.105 & 0.182 & 0.564 \\
\hline & & MANUFACTURA & 0.148 & 0.294 & 0.616 \\
\hline & \multirow[t]{2}{*}{ COMERCIO } & SERVICIO & -0.105 & 0.182 & 0.564 \\
\hline & & MANUFACTURA & 0.043 & 0.317 & 0.893 \\
\hline & \multirow[t]{2}{*}{ MANUFACTURA } & SERVICIO & -0.148 & 0.294 & 0.616 \\
\hline & & COMERCIO & -0.043 & 0.317 & 0.893 \\
\hline \multirow[t]{6}{*}{ OYT8 } & \multirow[t]{2}{*}{ SERVICIO } & COMERCIO & 0.204 & 0.202 & 0.314 \\
\hline & & MANUFACTURA & 0.297 & 0.327 & 0.364 \\
\hline & \multirow[t]{2}{*}{ COMERCIO } & SERVICIO & -0.204 & 0.202 & 0.314 \\
\hline & & MANUFACTURA & 0.093 & 0.352 & 0.791 \\
\hline & \multirow[t]{2}{*}{ MANUFACTURA } & SERVICIO & -0.297 & 0.327 & 0.364 \\
\hline & & COMERCIO & -0.093 & 0.352 & 0.791 \\
\hline \multirow[t]{6}{*}{ OYT9 } & \multirow[t]{2}{*}{ SERVICIO } & COMERCIO & 0.114 & 0.188 & 0.546 \\
\hline & & MANUFACTURA & 0.571 & 0.304 & 0.063 \\
\hline & \multirow[t]{2}{*}{ COMERCIO } & SERVICIO & -0.114 & 0.188 & 0.546 \\
\hline & & MANUFACTURA & 0.457 & 0.328 & 0.166 \\
\hline & \multirow[t]{2}{*}{ MANUFACTURA } & SERVICIO & -0.571 & 0.304 & 0.063 \\
\hline & & COMERCIO & -0.457 & 0.328 & 0.166 \\
\hline \multirow[t]{2}{*}{ OYT10 } & \multirow[t]{2}{*}{ SERVICIO } & COMERCIO & -0.069 & 0.192 & 0.718 \\
\hline & & MANUFACTURA & 0.037 & 0.310 & 0.906 \\
\hline
\end{tabular}




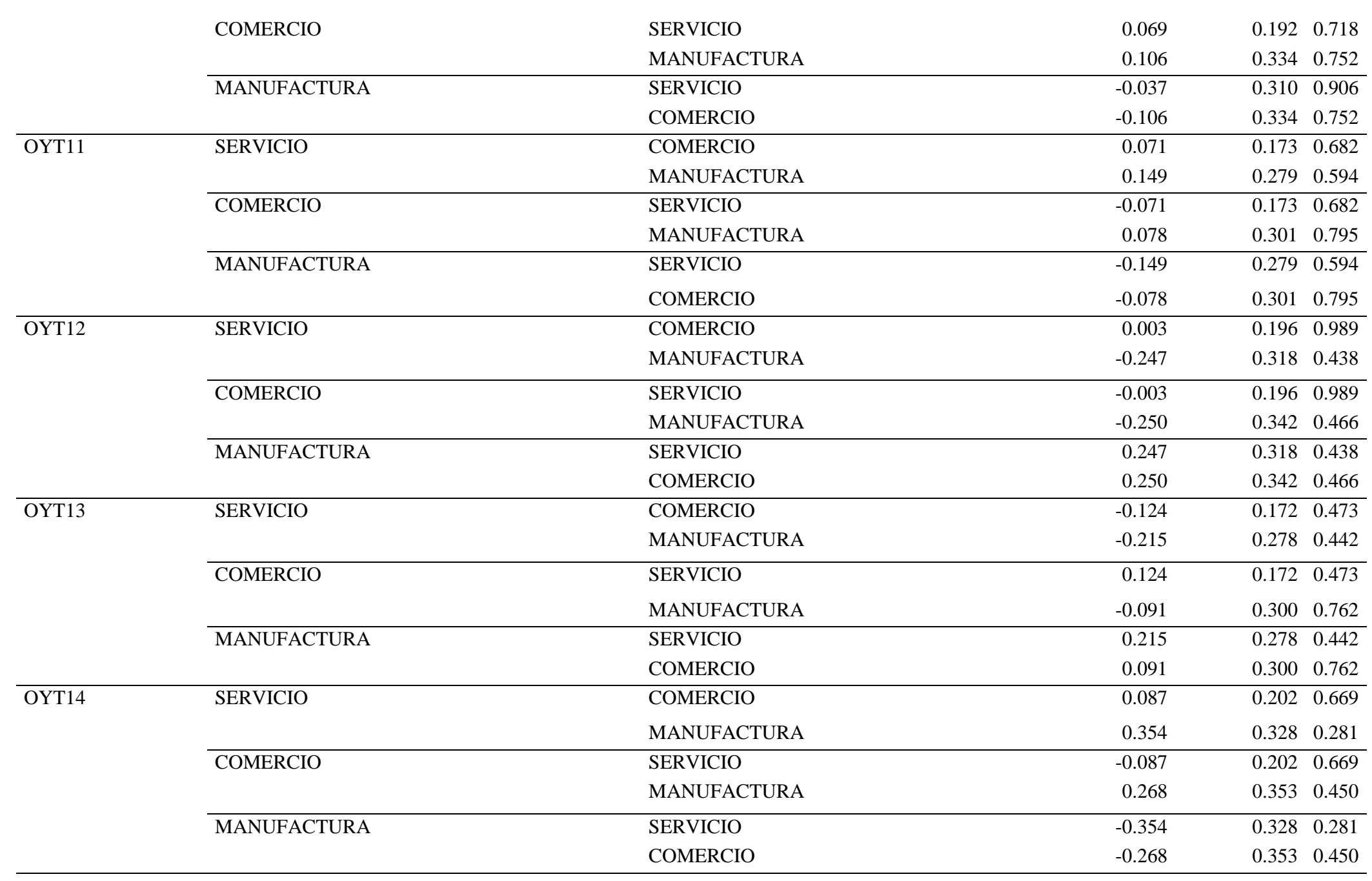

\section{1}

(2019) MLSER, 3(2), 7-32 
